\title{
Prácticas de mejora continua, con enfoque Kaizen, en empresas del Distrito Metropolitano de Quito: Un estudio exploratorio
}

\author{
Karla Alvarado RamíreziD, Víctor Pumisacho Álvaro iD \\ Escuela Politécnica Nacional (Ecuador) \\ karla.alvarado@epn.edu.ec, victor.pumisacho@epn.edu.ec
}

Received October, 2016

Accepted February, 2017

\section{Resumen}

Objeto: El propósito de este artículo es evaluar la práctica de mejora continua en medianas y grandes empresas de manufactura y servicios del Distrito Metropolitano de Quito (DMQ); examinar los beneficios y dificultades en el sostenimiento de la mejora continua; y, estudiar la participación de los diferentes niveles jerárquicos organizacionales en la práctica de mejora continua.

Diseño/metodología/enfoque: Se condujo un estudio exploratorio. Se seleccionaron medianas y grandes empresas de servicios y de manufactura del DMQ. Los métodos cualitativos utilizados para obtener los datos fueron: observación directa, análisis documental y entrevistas a profundidad semi-estructuradas.

Aportaciones y resultados: Los resultados muestran una preferencia por el uso de técnicas más sencillas para identificar y solucionar problemas como las siete herramientas básicas de calidad. Dentro de este artículo se describen a detalle los elementos potenciadores y las barreras que se presentan en el mantenimiento de la mejora continua. 
Limitaciones: Este artículo utiliza una metodología cualitativa, por lo que sus resultados no se pueden generalizar, sólo se pueden referenciar utilizando el contexto específico de las empresas estudiadas.

Implicaciones prácticas: El estudio pretende tener una aportación analítica. Los resultados evidencian beneficios económicos y para el recurso humano, considerando principalmente la minimización de procesos innecesarios y la oportunidad de desarrollo profesional que se ofrece al personal, datos importantes para aquellas personas involucradas en proyectos de mejora continua en empresas del DMQ.

Implicaciones sociales: Las conclusiones tienen importantes implicaciones para la investigación. La mejora continua debe tener en cuenta no sólo los beneficios económicos, sino también los factores humanos, mismos que pueden influir en la calidad de vida del trabajador.

Originalidad / Valor añadido: El trabajo presenta aportes empíricos a la literatura mediante la exposición del funcionamiento del término Kaizen en el contexto latinoamericano, Quito - Ecuador.

Palabras clave: Kaizen, Mejora continua, Técnicas, Herramientas, Prácticas

Códigos JEL: L23, M1, M10

Title: Continuous improvement practices with Kaizen approach in companies of the metropolitan district of Quito: An exploratory study

\section{Abstract}

Purpose: The purpose of this article is to evaluate the practice of continuous improvement in medium and large manufacturing companies and services of the Metropolitan District of Quito (DMQ); examine the benefits and difficulties in sustaining continuous improvement; and, to study the participation of the different hierarchical organizational levels in the practice of continuous improvement.

Design/methodology: An exploratory study was conducted. They were selected, medium and large companies, both manufacturing and services of the DMQ. Qualitative methods used to obtain the data were: direct observation, document analysis and interviews with semi-structured depth. 
Findings: The results show a preference for the use of simpler techniques to identify and solve problems such as the seven basic quality tools. Within this article describes in detail the enhancers and barriers that are presented in the maintenance of continuous improvement.

Research limitations/implications: This article uses a qualitative methodology, so their results cannot be generalized, can only be referenced using the specific context of the companies studied.

Practical implications: The study aims to have an analytical contribution. The results show economic benefits and for the human resource, considering mainly the minimization of unnecessary processes and the opportunity of professional development that is offered to the personnel, important data for those people involved in projects of continuous improvement in DMQ companies.

Social implications: The conclusions have important implications for research. Continuous improvement should take into account not only economic benefits but also human factors, same that can influence the quality of life of workers.

Originality/value: The paper presents empirical contributions for the literature by exposing of the implementation of the Kaizen concept in the Latin American context, Quito - Ecuador.

Keywords: Kaizen, Continuous improvement, Techniques, Tools, Practice

Jel Codes: L23, M1, M10

\section{Introducción}

En la actualidad, para alcanzar una posición competitiva en el mercado, las empresas apremian cambios organizacionales, apoyados en una cultura de mejora continua (Emiliani, 2005). En este sentido, el Kaizen involucra la participación de todos los actores del proceso (Imai, 1989), pues la práctica consiste en establecer un estándar, mantenerlo y, mejorarlo (Wittenberg, 1994). Es así que varios autores coinciden en tres nociones básicas del Kaizen: a) es continuo, b) es incremental; y, c) es participativo (Brunet \& New, 2003). Esto sugiere que el concepto de Kaizen comprende un proceso de actividades que se implementa continuamente y que asegura una constante búsqueda de la innovación. 
La mejora continua es asociada a con una diversidad de desarrollos organizacionales incluyendo la adopción de enfoques modernos como Gestión de la Calidad Total (TQM), Manufactura Esbelta, Teoría de Restricciones (TOC), Seis Sigma (SS), Kaizen, entre otros (Singh \& Singh, 2015). De esta manera, la gestión de la calidad total o TQM es un enfoque de gestión que involucra la mejora radical del proceso para obtener grandes resultados (Davenport \& Short, 1990). Por su parte, Womack y Jones (1996) describen lean manufacturing o manufactura esbelta como un enfoque sistémico para eliminar desperdicios respondiendo a la demanda de clientes, mediante la mejora continua en la búsqueda de la perfección. TOC desarrolla procedimientos para optimizar restricciones del sistema (Goldratt \& Cox, 1992). Así, la implementación de proyectos seis sigma (SS) a través del ciclo DMAIC busca reducir la variación en todos los procesos de la organización (Ehie \& Sheu, 2005, Linderman, Schroeder, Zaheer \& Choo, 2003; Breyfogle, 1999). Ante esto, varias organizaciones con el fin de mejorar sus resultados y aprovechar las ventajas que ofrece cada enfoque han fusionado diferentes iniciativas de mejora continua (Singh \& Singh, 2015). En los últimos años las organizaciones han mostrado mayor interés en la implementación de la mejora continua con enfoque Kaizen (Suárez-Barraza, 2007; Ortiz, 2009), enfatizando la consecución de una ventaja competitiva (Hammer, 2004), que permita mejorar la productividad y competitividad organizacional (Suárez-Barraza \& Miguel-Dávila, 2009), involucrando en ésta búsqueda la reducción de costos, tiempos y el logro de estándares de alta calidad, mediante el trabajo en equipo de todos los niveles organizacionales, esfuerzos que están orientados hacia la satisfacción de necesidades y expectativas de clientes internos y externos (Salgueiro, 1999; Alukal \& Manos, 2006; García, Dueñas, Rainusso, Diez \& García, 2010). Sin embargo, a pesar de esta búsqueda sostenida de la mejora continua, algunos estudios evidencian aspectos inhibidores que obstaculizan el cambio y que en muchos casos dificultan la aplicación de las mejoras organizacionales (Prajogo \& Sohal, 2004).

Por tal motivo, aunque existe una vasta literatura que expone la importancia del Kaizen y reconocen que la mejora continua juega un papel primordial en la administración (De Haan, Yamamoto \& Lovink, 2001; Brunet \& New, 2003) todavía hace falta explorar los resultados en la práctica. Escasos estudios exponen la conceptualización y aplicación del Kaizen en el contexto latinoamericano, y menos aún en el Ecuador. En consecuencia, el objetivo del presente estudio es:

- determinar la práctica de técnicas o herramientas de mejora continua, orientado al enfoque Kaizen, en medianas y grandes empresas de manufactura y servicios del Distrito Metropolitano de Quito (DMQ); 
- examinar los beneficios y dificultades en el sostenimiento de la mejora continua que presentan las organizaciones analizadas; $y$,

- estudiar la participación de los diferentes niveles jerárquicos en las organizaciones estudiadas.

\section{Fundamento teórico}

\section{1. ¿Qué es el Kaizen?}

El Kaizen como mejoramiento continuo involucra a todos, incluyendo a los gerentes y trabajadores por igual (Imai, 1989); en este sentido, el Kaizen busca mejorar la actitud de las personas, considerando que los esfuerzos generan mejores resultados en el largo plazo (Wittenberg, 1994; Bessant, Caffyn \& Gallagher, 2001). Así mismo, Imai (1997) sostiene que en el lugar de trabajo los empleados deben mantener y mejorar los niveles laborales con el fin de mejorar el rendimiento de cada proceso. Para Newitt (1996), el término Kaizen se deriva de dos elementos japoneses (Kanjis) que significan: Kai = Cambio y Zen $=$ Bueno (para mejorar); en definitiva, pequeñas mejoras realizadas como resultado de un esfuerzo continuo (Lillrank \& Kano, 1989, pp. 28). Bessant et al. (2001, pp. 68) han argumentado que gran parte de la literatura sobre mejora continua, la define como un conjunto de prácticas que pueden contribuir para que una organización mejore lo que actualmente realiza. Sawada (1995) sugiere que la forma de implementar este concepto es a través de equipos de personas conocido como equipos Kaizen o equipos de mejora continua de procesos.

\subsection{Técnicas, prácticas y herramientas gerenciales del Kaizen}

Kaizen es un proceso de resolución de problemas, en donde cualquier mejora debe ser estandarizada para alcanzar el siguiente nivel de mejora (Imai, 1986). Dado que cualquier producto y actividad es objeto de mejora, el Kaizen cubre diversas técnicas de gestión que han sido desarrollados durante los últimos años para hacer visible el problema. En la actualidad es de conocimiento amplio que gran parte de las técnicas de gestión de calidad, así como sus principios fueron transferidos por Deming y Juran (Imai, 1986; Lillrank, 1995). En la literatura se encuentra una diversidad de prácticas, técnicas y/o herramientas utilizadas, mismas que en una revisión extensa han sido agrupadas como se muestra en la Tabla 1. 


\begin{tabular}{|c|c|}
\hline Autor & Técnicas / Prácticas / Herramientas \\
\hline Imai (1989) & $\begin{array}{l}\text { Control total de la calidad, círculos de control de calidad, sistemas de } \\
\text { sugerencias, automatización, mantenimiento total productivo, Kanban, } \\
\text { mejoramiento de calidad, justo a tiempo, cero defectos, actividades en grupos } \\
\text { pequeños, relaciones cooperativas, mejoramiento de la productividad. }\end{array}$ \\
\hline Wittenberg (1994), Bond (1999) & $\begin{array}{l}\text { Círculos de calidad, calidad total, control, mantenimiento productivo total, } \\
\text { sistemas de sugerencias, Kanban, justo a tiempo, mejora de la productividad, } \\
\text { robótica, formación y automatización. }\end{array}$ \\
\hline $\begin{array}{l}\text { Nonaka (1995), Dankbaar (1997), } \\
\text { De Tréville y Antonakis (2006) }\end{array}$ & $\begin{array}{l}\text { Gestión del conocimiento: equipos multi-funcionales, selección y formación de } \\
\text { los empleados, participación de los trabajadores en programas de mejora } \\
\text { continua. }\end{array}$ \\
\hline $\begin{array}{l}\text { Ishikawa (1985), Liker(2004), Hino } \\
\text { (2006), Liker y Meier (2006), Van } \\
\text { Scyoc (2008), Suárez-Barraza y } \\
\text { Dávila (2011), Suárez-Barraza y } \\
\text { Ramis-Pujol (2012) }\end{array}$ & $\begin{array}{l}\text { Lean, 5S, teoría de las restricciones, Seis Sigma, historia de la calidad, equipos } \\
\text { de mejora, formación, rediseño de procesos, principios de calidad, proceso de } \\
\text { mejora continua (PDCA), estandarización, administración del sitio de trabajo, } \\
\text { talleres de mejora. }\end{array}$ \\
\hline Brunet y New (2003) & $\begin{array}{l}\text { Cero defectos, operación de sistemas de sugerencias, promoción de programas } \\
\text { y objetivos mediante la implicación de la dirección, círculos de calidad. }\end{array}$ \\
\hline
\end{tabular}

Tabla 1. Técnicas, prácticas y herramientas gerenciales en Kaizen (Elaboración propia a partir de la literatura existente)

\subsection{E1 Kaizen y la jerarquía administrativa}

La alta administración introduce el Kaizen como estrategia corporativa, proporcionando apoyo y dirección, estableciendo políticas, metas y construyendo sistemas, procedimientos y estructuras (Imai, 1989; Brunet \& New, 2003). En cambio, los gerentes de nivel medio, despliegan y ejecutan las metas, usan programas de entrenamiento, desarrollan habilidades, establecen, mantienen y mejoran los estándares (Imai, 1989; Wittenberg, 1994). Así, la primera línea, formula planes, apoya a los círculos de calidad y al sistema de sugerencias; y el nivel operativo, practica un autodesarrollo constante, ampliando las habilidades y el desempeño (Imai, 1989). En consecuencia, administradores, gerentes y empleados trabajan juntos en la identificación de posibles mejoras (Van Scyoc, 2008).

\subsection{E1 Kaizen y los resultados organizacionales}

El Kaizen se orienta a las personas, al mejoramiento de procesos y, a la satisfacción de clientes (Wittenberg, 1994; Oropesa, García, Maldonado \& Martínez, 2016) logrando en consecuencia beneficios cualitativos, cuantitativos económicos y humanos, que se ven reflejados en rendimientos y relaciones de clientes a través del ahorro de costos, reducción de tiempos de espera y de ciclos, mejora de la calidad, así como en la disminución de los accidentes de trabajo, todo esto gracias al desarrollo del recurso humano de la organización (Prajogo \& Sohal, 2004; Alukal \& Manos, 2006; García et al., 2010). 


\section{Metodología}

Estudios cualitativos que busquen responder a preguntas de ¿cómo? y ¿por qué? las prácticas del Kaizen, en el contexto latinoamericano y en el Ecuador son escasos. Dentro de estas limitaciones, en la presente investigación se desarrolló un estudio exploratorio, buscando comprender desde un ángulo cualitativo, la aplicación del Kaizen en el contexto organizacional, seleccionando medianas y grandes empresas tanto de servicios como de manufactura del Distrito Metropolitano de Quito (DMQ). Se decidió trabajar con empresas del DMQ, ya que de acuerdo al Servicio de Acreditación Ecuatoriano SAE, son las empresas capitalinas las que han tenido mayor demanda en certificaciones de calidad en Ecuador en los últimos años. Se remitió invitación a aproximadamente 70 empresas que podrían beneficiarse o estar interesadas en el estudio, de las cuales 33 se convirtieron en empresas participantes voluntarias de la investigación. El muestreo cualitativo teórico utilizado en la investigación pretende en todo momento la representación tipológica de acuerdo a los objetivos del estudio considerando los contingentes de medios y tiempo. Bajo este muestreo se levantaron categorías sobre las cuáles se buscó profundizar, entendiendo el fenómeno hasta llegar a la saturación y riqueza de datos. Las unidades se seleccionaron porque poseen las siguientes características:

- empresas medianas y/o grandes de manufactura y/o servicios que operen en el DMQ, siguiendo el criterio de muestreo teórico con la intención de explorar el concepto Kaizen (Glaser \& Strauss, 1967; Strauss \& Corbin, 1994; Ritchie \& Lewis, 2003); y,

- empresas que cuenten con al menos una certificación y/o práctica de calidad.

La adecuada selección de las empresas involucradas en un estudio resulta ser de gran importancia para el levantamiento de información en la investigación empírica. La clasificación de empresas se la realizó considerando dos criterios, en el siguiente orden:

- $\quad$ volumen de ventas anual; $y$,

- número de personas ocupadas, esto de acuerdo a la Clasificación emitida por la Comunidad Andina de Naciones (CAN), la cual establece como Mediana empresa a quien genera un volumen de ventas anual desde 1,000,001 a 5,000,000 y mantiene entre 50 a 199 empleados; y, Grande empresa, la cual genera un volumen de ventas anual de 5,000,001 en adelante y mantiene más de 200 empleados. 
Además se consideró el criterio de calidad, reconociendo que la certificación hace referencia a la aplicación de un sistema de calidad, y de acuerdo a las empresas es un reconocimiento avalado por una opinión técnica, mientras que las prácticas de calidad se refieren a experiencias exitosas en sistemas de gestión relacionados con la excelencia y la calidad total que se pueden replicar.

En el análisis de las empresas se utilizó técnicas de recolección de datos (Yin, 1994; Stake, 2000) como observación directa, entrevistas a profundidad semi-estructuradas (33 directores involucrados en la mejora continua - Revisar Tabla 2) y análisis documental, mismos que permitieron comprender el fenómeno de estudio. En la observación directa, se realizaron visitas a cada una de las empresas seleccionadas, observando los lugares de trabajo y recolectando material documental referido a las prácticas de mejora continua como sitios web, proyectos de mejora, formatos, reportes, etc. La evidencia documental completó el conjunto de datos.

\begin{tabular}{|c|c|c|c|c|c|c|}
\hline Clasificación & $\%$ & Tipología & $\%$ & Actividad económica & $\%$ & Puesto del informante \\
\hline \multirow{9}{*}{ Medianas } & \multirow{9}{*}{$73 \%$} & Manufactura & $25 \%$ & Industrias manufactureras & $100 \%$ & $\begin{array}{l}\text { Gerencia de una línea de } \\
\text { producción o jefe de planta }\end{array}$ \\
\hline & & \multirow{8}{*}{ Servicios } & \multirow{8}{*}{$75 \%$} & Construcción & $11 \%$ & $\begin{array}{l}\text { Gerencia, dirección o } \\
\text { jefatura técnica }\end{array}$ \\
\hline & & & & Comercio al por mayor y al por menor & $22 \%$ & $\begin{array}{l}\text { Gerencia, dirección o } \\
\text { jefatura técnica }\end{array}$ \\
\hline & & & & Transporte y almacenamiento & $6 \%$ & $\begin{array}{l}\text { Gerencia, dirección o } \\
\text { jefatura técnica }\end{array}$ \\
\hline & & & & $\begin{array}{l}\text { Actividades de alojamiento y de } \\
\text { servicio de comidas }\end{array}$ & $11 \%$ & Coordinación de calidad \\
\hline & & & & Actividades financieras y de seguros & $17 \%$ & $\begin{array}{l}\text { Gerencia, dirección o } \\
\text { jefatura técnica }\end{array}$ \\
\hline & & & & $\begin{array}{l}\text { Actividades profesionales, científicas y } \\
\text { técnicas }\end{array}$ & $6 \%$ & Coordinación de calidad \\
\hline & & & & $\begin{array}{l}\text { Actividades de servicios } \\
\text { administrativos y de apoyo }\end{array}$ & $6 \%$ & Coordinación de calidad \\
\hline & & & & Enseñanza & $22 \%$ & Coordinación de calidad \\
\hline \multirow{5}{*}{ Grandes } & \multirow{5}{*}{$27 \%$} & Manufactura & $44 \%$ & Industrias manufactureras & $100 \%$ & $\begin{array}{l}\text { Gerencia de una línea de } \\
\text { producción o jefe de planta }\end{array}$ \\
\hline & & \multirow{4}{*}{ Servicios } & \multirow{4}{*}{$56 \%$} & Comercio al por mayor y al por menor & $40 \%$ & $\begin{array}{l}\text { Gerencia, dirección o } \\
\text { jefatura técnica }\end{array}$ \\
\hline & & & & Transporte y almacenamiento & $20 \%$ & $\begin{array}{l}\text { Gerencia, dirección o } \\
\text { jefatura técnica }\end{array}$ \\
\hline & & & & $\begin{array}{l}\text { Actividades de alojamiento y de } \\
\text { servicio de comidas }\end{array}$ & $20 \%$ & Coordinación de calidad \\
\hline & & & & Enseñanza & $20 \%$ & Coordinación de calidad \\
\hline
\end{tabular}

Tabla 2. Caracterización del grupo de empresas involucradas en el estudio. (Elaboración propia a partir de la clasificación de empresas emitida por la CAN, código CIIU 4.0 (Clasificación Nacional de Actividades Económicas) e información de la empresa) 
Finalmente, con el propósito de incrementar la confiabilidad del estudio se llevó a cabo un protocolo de entrevista (Pettigrew, 1997) buscando la construcción conjunta de significados respecto al tema objeto de estudio (Janesick, 1998). Tras el consentimiento de confidencialidad se procedió a la grabación del audio para el levantamiento de información, por cada organización se entrevistó al director general o al responsable del programa de mejora continua, o del área de calidad (Philips, 1981). Esta actividad fue llevada a cabo por dos entrevistadores; en total se realizaron 33 entrevistas en los meses de abril a julio de 2016, siguiendo estrictamente el protocolo de investigación, pero dando flexibilidad a ciertas contestaciones significativas para la temática. Cada entrevista tuvo una duración aproximada de 30 a 40 minutos y buscó comprender las técnicas/herramientas que sustentan la filosofía Kaizen en las empresas de estudio. El protocolo de la entrevista estuvo dividido en cinco partes:

- la comprensión de la práctica de mejora continua (Kaizen) de la empresa,

- las prácticas gerenciales de mejora continua aplicadas en la empresa,

- las barreras en la aplicación de mejora continua,

- los beneficios ligados a la mejora continua; y,

- la participación del talento humano en la mejora continua.

El análisis comenzó con la codificación abierta, determinando posteriormente etiquetas de códigos y categorías. Los códigos y categorías fueron clasificados, comparados y verificados hasta su saturación, es decir, hasta que el análisis no presentará nuevos códigos (Neuman, 2009), de esta manera, el estudio buscó en todo momento desarrollar códigos y categorías en lugar de imponer clasificaciones (Glaser, 1978). Finalmente debe mencionarse que en el análisis cualitativo se trató de asegurar la validez del estudio a través de la utilización de diferentes fuentes de información y estableciendo una cadena planificada de recolección de datos. 


\section{Resultados}

Los resultados de esta investigación cualitativa han sido agrupados en cinco temas principales, mismos que pasan a ser descritos a continuación y explican la situación actual global de las organizaciones en relación al tópico de investigación. Dadas las características de la muestra de la Tabla 2, la aplicación del enfoque de mejora continua en las empresas del DMQ, es baja; lo que sugiere que varias organizaciones en el afán de implementar diferentes actividades que permitan la mejora de la calidad en tiempos cortos dejan de lado el desarrollo sistemático y a largo plazo de las estrategias que posibilitan alcanzar una cultura de mejora sostenida, orientada a la eliminación de desperdicios en los procesos y sistemas organizacionales que incluyan la participación de los trabajadores en todos los niveles de jerarquía operacional. Los resultados muestran una preferencia por el uso de técnicas más sencillas para identificar y solucionar problemas tales como las siete herramientas básicas de calidad.

\subsection{Comprensión de la Práctica de Mejora Continua (Kaizen)}

En las empresas objeto de estudio se determinó que un $85 \%$ conoce la práctica como Mejora Continua desconociendo el término Kaizen, y un 15\% propiamente como Kaizen, siendo un grupo de las grandes empresas las que reconocen este término. Ante esta comprensión, los directivos mencionan:

- la aplicación se conoce como mejora continua, desconociendo el término Kaizen.

- la práctica de mejora continua se la lleva a cabo considerando la evaluación de actividades que aportan valor y satisfacción de los requerimientos de los clientes.

- los procesos de mejora continua se basan en la norma ISO y en los planes estratégicos.

- la aplicación se lleva a cabo como ciclo de mejora continua - PDCA.

En este sentido, en el contexto ecuatoriano del DMQ, se evidencia que el término japonés ha sido incorporado mediante la comprensión del mejoramiento continuo en los procesos organizacionales, mismo que permite alcanzar satisfacción de los clientes, lo que se ve reflejado en beneficios económicos para la organización. Esto muestra como la comprensión del concepto se alinea a criterios económicos y humanos expuestos en la literatura académica (Wittenberg, 1994; Prajogo \& Sohal, 2004; Alukal \& Manos, 2006; García et al., 2010; Oropesa et al., 2016). 


\subsection{Prácticas Gerenciales}

El porcentaje de las prácticas gerenciales aplicadas y los resultados exponen que las herramientas de calidad son las más utilizadas como se observa en la Figura 1.

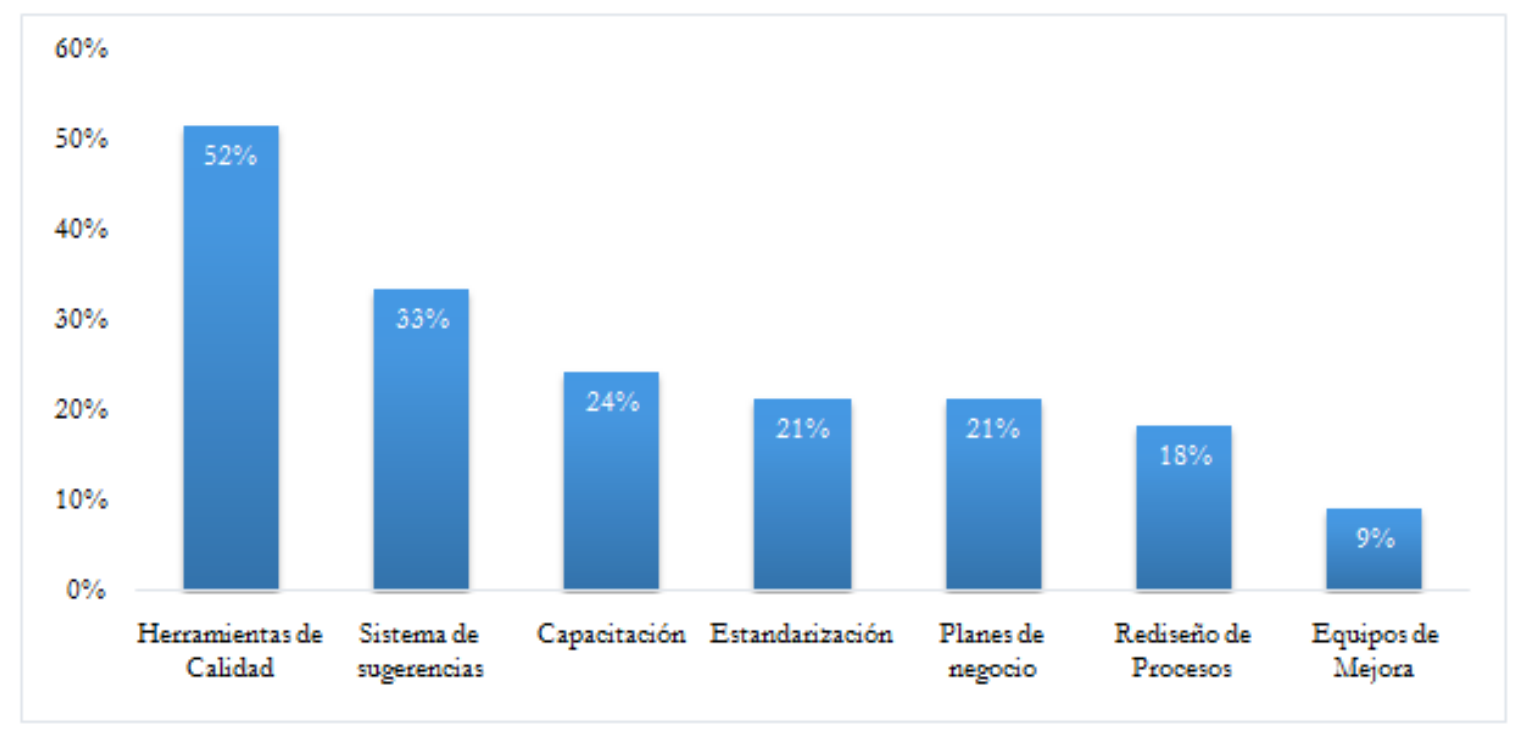

Figura 1. Prácticas Gerenciales de Mejora Continua. Información recopilada en la entrevista

La literatura existente expone diferentes prácticas gerenciales y las empresas de estudio así lo confirman, el producto de la investigación muestra un mayor énfasis en el uso de herramientas de calidad (52\%) y en el manejo de sistemas de sugerencias (33\%), prácticas que se vinculan a la mejora continua del trabajo diario (Suárez-Barraza \& Ramis-Pujol, 2012), buscando solucionar problemas del lugar de trabajo de una manera constante y diaria.

Como parte del enfoque hacia las personas (De Tréville \& Antonakis, 2006; Brunet \& New, 2003), se tiene la presencia de la educación y capacitación (24\%) y la creación de equipos de mejora (9\%), sin embargo a pesar de la existencia de los equipos de mejora estos aún presentan una estructura informal. El enfoque hacia los elementos básicos para la implementación del Kaizen (Imai, 1989; Liker \& Meier, 2006) se ve reflejado en la estandarización (21\%); mientras que el mantenimiento y mejora de los estándares se lo evidencia parcialmente en la aplicación del ciclo PDCA, mediante los planes de negocio (21\%), en el uso de ciertos proyectos de mejora. Las metodologías restantes tienen menos participación porcentual y están vinculadas al enfoque de procesos (18\%). 
Considerando las tres prácticas más significativas del estudio, en lo que concierne a técnicas y herramientas de la filosofía Kaizen, (ver Figura 2), se observa el uso de algunas herramientas antiguas y nuevas de calidad como el diagrama de Ishikawa, los diagramas de flujo, la lista de verificación o “check list', y el árbol de problemas. En los sistemas de sugerencias se usan técnicas como el OPV (otro punto de vista) y el método $5 \mathrm{~W}$, mediante reuniones semanales mantenidas en las distintas empresas objeto de estudio; sin embargo, cabe señalar que estas herramientas no se utilizan en el contexto de la técnica de la historia de la calidad (QC Story) como lo propone Imai (1989) y más bien se consideran como esfuerzos aislados poco centrados en la filosofía de mejora.

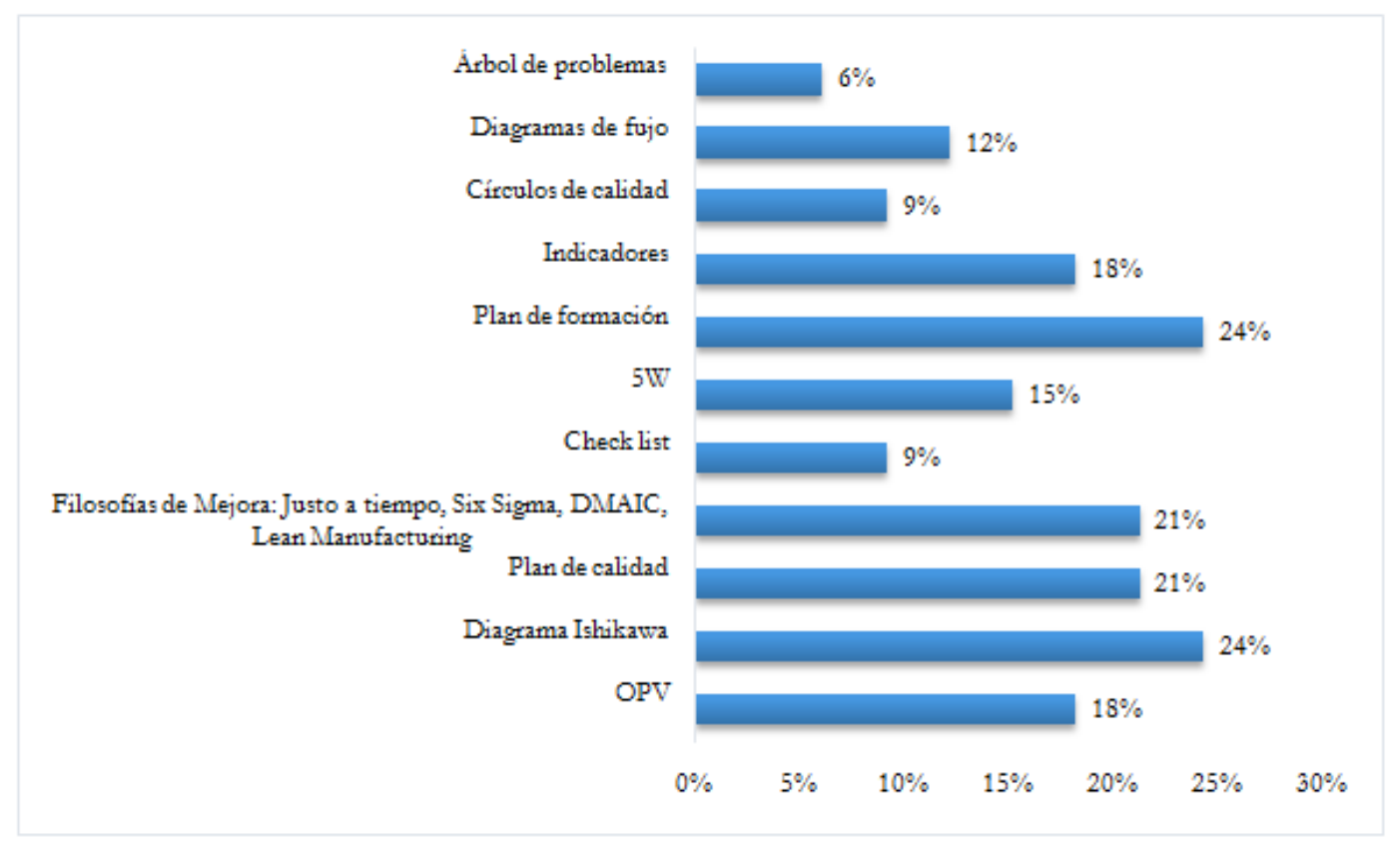

Figura 2. Herramientas y Técnicas Gerenciales de Mejora Continua. Información recopilada en la entrevista

\subsection{Barreras o inhibidores en la aplicación de mejora continua}

Las principales barreras encontradas en la aplicación de la mejora continua detectadas en el estudio se muestran en la Tabla 3. Se consideran la resistencia al cambio $(79 \%)$ y el bajo entendimiento de la metodología Kaizen (61\%) como las causas más fuertes del abandono de prácticas, herramientas o técnicas de mejoramiento continuo. Frente a la resistencia al cambio, el estudio muestra un conflicto entre el enfoque de mejora continua y la cultura organizacional actual de las organizaciones objeto de estudio. El bajo entendimiento del Kaizen desde los mandos altos hasta los mandos bajos, al no tener claro el propósito de mejora y realizar esfuerzos aislados, provoca fallos en el cumplimiento de proyectos. 


\begin{tabular}{|l|r|}
\hline \multicolumn{1}{|c|}{ Inhibidores } & \% \\
\hline Resistencia al cambio (costumbre, temor, etc.) & $79 \%$ \\
\hline Bajo entendimiento del Kaizen & $61 \%$ \\
\hline Falta de compromiso formal y apoyo de la alta dirección & $55 \%$ \\
\hline Restricción de recursos (tiempo, dinero y personas) & $55 \%$ \\
\hline Falta de involucramiento de todo el personal & $55 \%$ \\
\hline Insuficiente experiencia en Kaizen por los equipos de trabajo & $42 \%$ \\
\hline Resultados visibles a largo plazo & $36 \%$ \\
\hline Desconocimiento de nuevos procesos & $12 \%$ \\
\hline Falta de motivación de los empleados a participar en las mejoras & $12 \%$ \\
\hline Falta de integración de los sistemas & $12 \%$ \\
\hline Enfoque exclusivo en la rentabilidad & $6 \%$ \\
\hline Pobre liderazgo & $6 \%$ \\
\hline Falta de seguimiento constante a las herramientas y técnicas Kaizen & $6 \%$ \\
\hline Centralización de decisiones & $6 \%$ \\
\hline Falta de empoderamiento & $6 \%$ \\
\hline
\end{tabular}

Tabla 3. Barreras en la aplicación del Kaizen. Información recopilada en la entrevista

\subsection{Beneficios ligados a la mejora continua}

Analizando desde un enfoque económico, el estudio refleja que la minimización de procesos innecesarios apoyadas en la tecnología digital y la comunicación empleado-cliente directo han permitido alcanzar un incremento de la eficiencia operativa (Wittenberg, 1994; Oropesa et al., 2016). Por otro lado, en términos de gestión humana, gran parte de las empresas coinciden en que el Kaizen apoya al desarrollo del personal, potencializando habilidades y destrezas del talento humano de la organización (Prajogo \& Sohal, 2004; Alukal \& Manos, 2006; García et al., 2010). Los beneficios más representativos que han logrado las organizaciones tras la aplicación de diferentes prácticas de mejora continua se ven reflejadas en la Tabla 4, estos beneficios consideran el aspecto económico y el humano.

\begin{tabular}{|l|r|}
\hline \multicolumn{1}{|c|}{ Beneficios } & $\mathbf{\%}$ \\
\hline Minimización de procesos & $64 \%$ \\
\hline Mejores relaciones y servicio para los clientes & $36 \%$ \\
\hline Prestigio, crecimiento y competitividad organizacional & $33 \%$ \\
\hline Incremento en ventas & $30 \%$ \\
\hline Disminución de costos y tiempos & $27 \%$ \\
\hline Mejora de habilidades de trabajadores & $27 \%$ \\
\hline Mejores índices de productividad y calidad & $21 \%$ \\
\hline Cumplimiento de normativas & $21 \%$ \\
\hline Reducción de excesos de inventarios & $15 \%$ \\
\hline Motivación de personal & $9 \%$ \\
\hline Mayor comunicación & $6 \%$ \\
\hline Mejora en el desempeño & $3 \%$ \\
\hline
\end{tabular}

Tabla 4. Beneficios tras la aplicación de Mejora Continua. Información recopilada en la entrevista 


\subsection{Participación del Talento Humano en la mejora continua}

La filosofía Kaizen implica el involucramiento de todo el personal de la organización (Imai, 1989), en este sentido en la Figura 3 se muestra el porcentaje de personas involucradas en la aplicación de la mejora continua. La participación de los mandos altos, referente a los directivos de la organización es baja (27\%), el mayor involucramiento se evidencia en los mandos bajos o personal de línea de la organización (58\%), apoyados por los mandos medios o supervisores (45\%) de las diversas empresas entrevistadas. Sin embargo, estos datos evidencian la falta de involucramiento de la alta dirección en la aplicación y comprensión de la mejora continua, tal como se evidenció en la parte cualitativa de esta investigación, disminuyendo la creación de redes de aprendizaje que promuevan cambios beneficiosos en la organización.

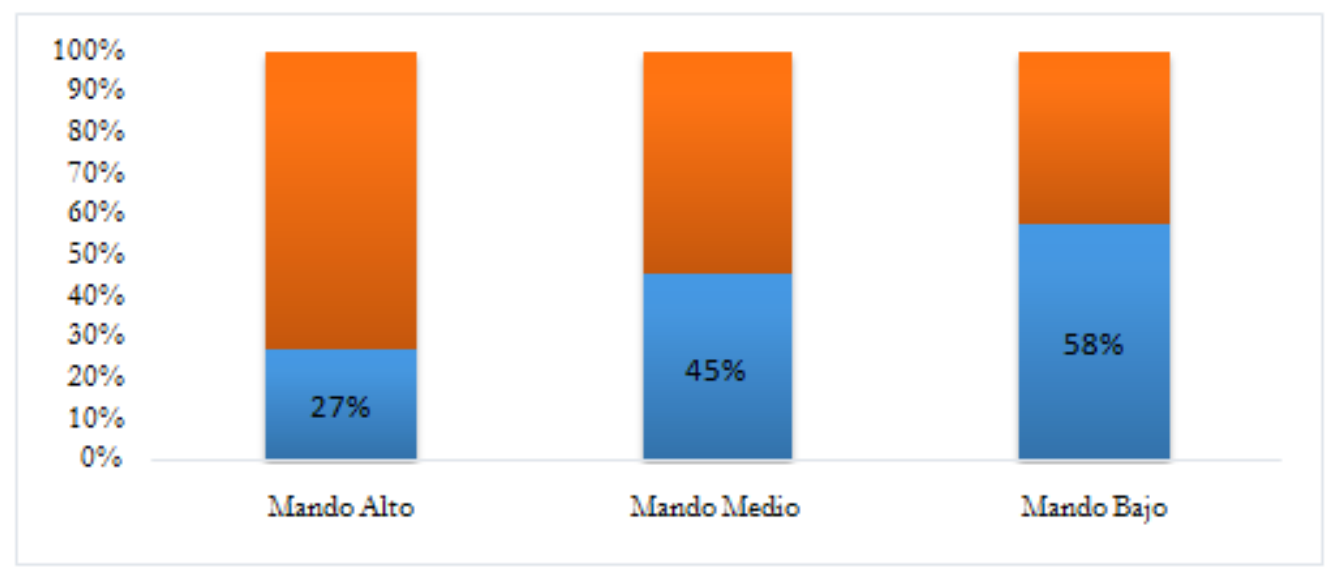

Figura 3. Participación del factor humano en la filosofía de mejora continua. Información recopilada en la entrevista

Dado que las certificaciones en calidad son decisiones voluntarias de las empresas, las empresas en estudio, como práctica de mejoramiento de la calidad, usan esquemas locales propios que invitan a que las sugerencias de mejora sean presentadas por los trabajadores, siguiendo un enfoque de abajo hacia arriba, es decir alentando el desarrollo de los empleados, generando oportunidades de crecimiento y recompensando las sugerencias de mejora. De esta manera, los directivos de estas organizaciones consideran que mediante las mejoras graduales presentadas por los mandos bajos y apoyadas por lo mandos medios, la gestión de la calidad total podrá alcanzarse en el largo plazo. 


\section{Conclusiones y discusión}

$\mathrm{Al}$ analizar la aplicación del Kaizen en las empresas del DMQ se evidencia una diversidad de formas en las que este concepto puede ser interpretado. Aún así, dos cuestiones importantes surgen del presente trabajo de investigación. La primera tiene que ver con el involucramiento de directivos, gerentes y trabajadores en la mejora continua, desarrollando un estado de aprendizaje continuo (Liker, 2004), demostrando cómo el trabajo en equipo puede resolver los problemas y superar las dificultades, contribuyendo así al logro de los objetivos organizacionales. En segundo lugar, el uso de técnicas, prácticas y/o herramientas como parte inherente al sistema de administración y operación de las empresas objeto de estudio, manteniendo y mejorando los estándares como resultado de esfuerzos progresivos, planteándose como reto para estas organizaciones el logro de la sostenibilidad de la mejora en el tiempo.

Estas cuestiones tienen implicaciones importantes para la investigación, ya que la mejora continua debe considerar no solo el beneficio económico, sino también el humano. Entonces, en términos académicos, se abre la necesidad de estudiar las prácticas de gestión de mejora continua considerando el ambiente económico y cultural cambiante, como lo expresan Lillrank (1995) y Brunet y New (2003) en sus investigaciones, esto debido a que la transferencia de prácticas gerenciales es compleja y requiere un análisis profundo en el contexto aplicado. Además, las lecciones apuntan a trabajar temas desde la perspectiva humana como la motivación y el aprendizaje continuo de la fuerza de trabajo en el sistema Kaizen, ya que al ser los empleados el soporte clave para la mejora continua (Recht \& Wildero m, 1998) se hace necesario comprender el desempeño organizacional mediante la inversión del esfuerzo individual y grupal.

En el estudio se evidencian limitaciones como el tamaño de la muestra, la zona geográfica seleccionada, las características propias del estudio como clasificación, tipología y actividad económica de las empresas, tipo de certificación o práctica de calidad empleada, por lo que próximos estudios pueden considerar otros enfoques donde la necesidad de mejora sea introducida por la alta dirección y la gestión de la calidad total se centre en mejoras a largo plazo. Así, los empresarios deben considerar que el compromiso de la alta dirección y el trabajo en equipo son los factores críticos dentro de la mejora continua, para lo cual el fomento de la creatividad y la resolución de problemas resultan ser elementos potenciadores que posibilitan una cultura de mejora sostenible. Ante esto, las empresas deben propiciar capacitación y/o formación de todos los miembros del equipo de trabajo, adoptando nuevas herramientas que posibiliten la mejora organizacional en el tiempo. 
Finalmente, los investigadores estamos conscientes que al usar una metodología cualitativa, los resultados y conclusiones no pueden generalizarse, sólo se pueden referenciar utilizando el contexto específico señalado. En este sentido, el presente estudio busca brindar un aporte analítico, mismo que puede ser desarrollado posteriormente en nuevas investigaciones enfocadas a otros contextos con la finalidad de poder desarrollar comparaciones. Además, estos hallazgos resultan útiles, pues abre un abanico de posibles prácticas que se pueden emplear en búsqueda del Kaizen para aquellos gestores que pretendan iniciar o se encuentren inmersos en esfuerzos de mejora continua.

\section{Referencias}

Alukal, G., \& Manos, A. (2006). Lean Kaizen: A simplified approach to process improvements. Milwaukee: ASQ Quality Press.

Bessant, J., Caffyn, S., \& Gallagher, M. (2001). An evolutionary model of continuous improvement behavior. Technovation, 21(2), 67-77. https://doi.org/10.1016/S0166-4972(00)00023-7

Breyfogle, F. III (1999). Implementing Six Sigma: Smarter Solution Using Statistical Methods. New York, NY: John Wiley \& Sons, Inc.

Bond, T.C. (1999). The role of performance measurement in continuous improvement. International Journal of Operations \& Production Management, 19(2), 1318-1334. https://doi.org/10.1108/01443579910294291

Brunet, A.P., \& New, S. (2003). Kaizen in Japan: An empirical study. International Journal of Operations \& Production Management, 23(12), 1426-1446. https://doi.org/10.1108/01443570310506704

Dankbaar, B. (1997). Lean production: Denial, confirmation or extension of socio-technical systems design?. Human Relations, 50(5), 567-583. https://doi.org/10.1023/A:1016991803180

Davenport, T., \& Short, J. (1990). The new industrial engineering: Information technology and business process redesign. Sloan Management Review, 31 (4), 11-27.

De Haan, J., Yamamoto, M., \& Lovink, G. (2001). Production planning in Japan: Rediscovering lost experiences or new insights?. International Journal of Production Economics, 71(1-3), 101-109. https://doi.org/10.1016/S0925-5273(00)00110-9

De Tréville, S., \& Antonakis, J. (2006). Could lean production job design be intrinsically motivating? Contextual, configurational, and levels-of-analysis issues. Journal of Operations Management, 24(2), 99-123. https://doi.org/10.1016/j.jom.2005.04.001 
Ehie, I., \& Sheu, C. (2005), Integrating six sigma and theory of constraints for continuous improvement: a case study. Journal of Manufacturing Technology Management, 16(5), 542-553. https://doi.org/10.1108/17410380510600518

Emiliani, M.L. (2005). Using kaizen to improve graduate business school degree programs. Quality Assurance in Education, 13(1), 37-52. https://doi.org/10.1108/09684880510578641

García, C., Dueñas, R., Rainusso, M., Diez, E., \& García, J. (2010). Sostenibilidad de los sistemas de mejora continua en la industria: Encuesta en la Comunidad Autónoma Vasca y Navarra. Intangible Capital, 6(1), 51-77.

Glaser, B. (1978). Theoretical sensitivity. California, Mill Valler. CA: Sociology Press.

Glaser, B.G. \& Strauss, A.L. (1967). The discovery of Grounded Theory: Strategies for Qualitative Research. New York: Aldine de Gruyter.

Goldratt, E., \& Cox, J. (1992). The Goal (2nd revised ed.). Croton-on-Hudson, NY: The North River Press.

Hammer, M. (2004). Deep Change. How Operational Innovation Can Transform your Company. Harvard Business Review, 82(4), 84-93. https://doi.org/10.1109/emr.2004.25106

Hino, S. (2006). Inside the Mind of Toyota. New York: Productivity Press.

Imai, M. (1986). Kaizen: The Key to Japan's Competitive Success. New York: McGraw-Hill.

Imai, M. (1989). Kaizen: La clave de la ventaja competitiva japonesa. México: Grupo Editorial Patria.

Imai, M. (1997). Gemba Kaizen: A Commonsense Low-cost Approach to Management. New York: McGraw-Hill. Ishikawa, K. (1985). What is Total Quality Control? The Japanese Way. Englewood Cliffs, NJ: Prentice-Hall. Janesick, V. J. (1998). Stretching exercises for qualitative researchers. Thousand Oaks, CA: Sage.

Liker, J. (2004). The Toyota Way. New York: Simon \& Schuster Inc.

Liker, J., \& Meier, D. (2006). The Toyota Way Fieldbook. New York: McGraw-Hill.

Lillrank, P. (1995). The transfer of management innovations from Japan. Organization Studies, 16(6), 971-989. https://doi.org/10.1177/017084069501600603

Lillrank, P., \& Kano, N. (1989). Continuous Improvement-Quality Control Circles in Japanese Industry. The Journal of Asian Studies, 50(2), 416-418. https://doi.org/10.2307/2057250

Linderman, K., Schroeder, R., Zaheer, S., \& Choo, A. (2003). Six sigma: A goal - theoretic perspective. Journal of Operations Management, 21(2), 193-203. https://doi.org/10.1016/S0272-6963(02)00087-6 
Neuman, L. (2009). Understanding research. Boston: Pearson Education.

Newitt, D.J. (1996). Beyond BPR \& TQM - Managing through processes: Is kaizen enough?. Institution of Electric Engineers (Eds.). London, U.K: Industrial Engineering. https://doi.org/10.1049/ic:19960785

Nonaka, I. (1995). The recent history of managing for quality in Japan. In J. Juran (Ed.), A History of Managing for Quality (pp. 517-552). Milwaukee: ASQC Quality Press.

Oropesa Vento, M., Garcia Alcaraz, J.L., Maldonado M., Aidé A., \& Martínez Loya, V. (2016). The impact of managerial commitment and Kaizen benefits on companies. Journal of Manufacturing Technology Management, 27(5), 692-712. https://doi.org/10.1108/JMTM-02-2016-0021

Ortiz, C. (2009). Kaizen and Kaizen event implementation. New York: Prentice-Hall.

Pettigrew, A.M. (1997). What is a processual analysis?. Scandinavian Journal of Management, 13(4), 337-348. https://doi.org/10.1016/S0956-5221(97)00020-1

Prajogo, D.I. \& Sohal, A.S. (2004). The sustainability and evolution of quality improvement Programmes- an Australian case study. Total Quality Management and Business Excellence, 15(2), 205-220. https://doi.org/10.1080/1478336032000149036

Philips, J. (1981). Assessing measurement error in key informant reports: A methodological note on organizational analysis in marketing. Journal of Marketing Research, 22(5), 395-415. https://doi.org/10.2307/3151333

Recht, R., \& Wilderom, C. (1998). Kaizen and culture: On the transferability of Japanese suggestion systems. International Business Review, 7(1), 7-22. https://doi.org/10.1016/S0969-5931(97)00048-6

Ritchie, J., \& Lewis, J. (2003). Qualitative Research Practice. London: Sage Publications.

Salgueiro, A. (1999). Cómo Mejorar los Procesos y la Productividad. Madrid: Asociación Española de Normalización y Certificación, AENOR.

Sawada, N. (1995). The Kaizen at Toyota production system. Quality Control Course, 6, 1-38. Nagoya: CHU-SAN-REN.

Singh, J., \& Singh, H. (2015). Continuous improvement philosophy - literature review and directions. Benchmarking: An International Journal, 22 (1), 75-119. https://doi.org/10.1108/BIJ-06-2012-0038

Stake, R. (2000). The case study method in social inquiry. In R. Gomm, M. Hammersley \& P. Foster (eds.), Case Study Method. New York: Sage Publications.

Strauss, A., \& Corbin, J. (1994). Grounded theory methodology: An overview. In N. Denzin, N. \& Y. Lincoln (Eds), Handbook of Qualitative Research (pp. 273-285). Thousand Oaks, CA: Sage. 
Suárez-Barraza, M.F. (2007). El Kaizen: La filosofía de Mejora Continua e Innovación Incremental detrás de la Administración por Calidad Total. México: Panorama Editorial.

Suárez-Barraza, M.F., \& Dávila, J. (2011). Implementación del Kaizen en México: Un estudio exploratorio de una aproximación gerencial japonesa en el contexto latinoamericano. Revista: INNOVAR. Revista de Ciencias Administrativas y Sociales, 21(41), 19-37.

Suárez-Barraza, M.F., \& Miguel-Dávila, J.A. (2009). En la búsqueda de un Espacio de Sostenibilidad: Un estudio empírico de la aplicación de la Mejora Continua de Procesos en Ayuntamientos Españoles. INNOVAR Journal of Administrative and Social Sciences, 19(35), 47-64.

Suárez-Barraza, M.F., \& Ramis-Pujol, J. (2012). An exploratory study of 5S: A multiple case study of multinational organizations in Mexico. Asian Journal on Quality, 13(1), 77-99.

https://doi.org/10.1108/15982681211237842

Van Scyoc, K. (2008). Process safety improvement: Quality and target zero. Journal of Hazardous Materials, 159(1), 42-48. https://doi.org/10.1016/j.jhazmat.2008.02.036

Wittenberg, G. (1994). Kaizen-The many ways of getting better. Assembly Automation, 14(4), 12-17. https://doi.org/10.1108/EUM0000000004213

Womack, J., \& Jones, D. (1996). Lean Thinking. New York, NY: Simon and Schuster.

Yin, R. (1994). Case Study Research, Design and Methods. Thousands Oaks, California: Sage Publications.

Intangible Capital, 2017 (www.intangiblecapital.org) 